\title{
IncRNA SNHG7 affects malignant tumor behaviors through downregulation of EZH2 in uveal melanoma cell lines
}

\author{
XUE WU, YIQUN YUAN, RUIQI MA, BINBIN XU and RUI ZHANG \\ Department of Ophthalmology, Eye and ENT Hospital of Fudan University, Shanghai 200031, P.R. China
}

Received July 4, 2019; Accepted November 22, 2019

DOI: 10.3892/ol.2019.11240

\begin{abstract}
Previous studies have demonstrated that the long non-coding RNA, small nucleolar RNA host gene 7 (SNHG7) plays an important role in several types of cancer; however, its role in the development of uveal melanoma (UM) remains unclear. The present study investigated the effect of SNHG7 on the prognosis of UM, as well as on cell proliferation, cell cycle and apoptosis of UM cell lines. Furthermore, the present study aimed to determine the molecular mechanisms underlying these effects. The association between SNHG7 and prognosis of UM was analyzed using detailed SNHG7 mRNA expression data and clinical information from The Cancer Genome Atlas database. Reverse transcription-quantitative PCR was used in order to detect the differential expression of SNHG7 in UM tissues and cell lines. Cell proliferation was detected using Cell Counting Kit-8 assays, following overexpression of SNHG7. A cell cycle assay was performed using propidium iodide/RNase staining. An apoptosis assay was performed using the Annexin-V-Fluorescein isothiocyanate apoptosis detection kit. The expression of enhancer of zeste homolog 2 (EZH2) was measured via western blotting. The results of the present study indicated that low expression of SNHG7 was associated with poor prognosis. Furthermore, increasing the expression of SNHG7 inhibited the proliferation of UM cells, suppressed cell cycle progression and promoted apoptosis. Western blot analysis results revealed that overexpression of SNHG7 downregulated EZH2 protein expression levels in
\end{abstract}

Correspondence to: Dr Rui Zhang, Department of Ophthalmology, Eye and ENT Hospital of Fudan University, 83 Fen Yang Road, Shanghai 200031, P.R. China

E-mail: zhangrui936@163.com

Abbreviations: lncRNA, long non-coding RNA; SNHG7, small nucleolar RNA host gene 7; UM, uveal melanoma; TCGA, The Cancer Genome Atlas; GEPIA, Gene Expression Profiling Interactive Analysis; OS, overall survival; RT-qPCR, reverse transcription-quantitative polymerase chain reaction; PBS, phosphatebuffered saline; RIPA, radioimmunoprecipitation assay buffer; EZH2, enhancer of zeste homolog 2; SNHG7+, SNHG7 overexpression group; NC, empty vector group; CCK-8, Cell Counting Kit-8

Key words: SNHG7, UM, lncRNA, EZH2, tumor suppressor
UM cell lines. The results of the present study demonstrated that SNHG7 inhibited malignant transformation of UM cells by regulating EZH2 expression.

\section{Introduction}

Uveal melanoma (UM) is the most common intraocular malignant tumor in adults worldwide (1). Primary UM can be treated by surgery with a low local recurrence rate. However, certain patients develop distant metastasis, predominantly in the liver, following primary tumor treatment (2). The development of distant metastasis is associated with a high mortality rate in up to half of the patients $(3,4)$. To date, the treatment of metastatic UM remains controversial (5). Thus, in-depth studies on the pathogenesis of UM are required for effective diagnosis and treatment, and improved prognosis.

Long non-coding RNA (lncRNA) is defined as a transcript $>200$ nucleotides long that lacks protein-coding potential (6), which is involved in various biological processes (such as chromatin remodeling, mRNA splicing, mRNA editing and translation) (7) and can be regulated through a number of different molecular mechanisms (8). A number of studies have demonstrated that lncRNAs play a key role in the occurrence and development of different types of tumor (9-11). Studies have demonstrated that lncRNAs regulate several cancer characteristics, including cell proliferation, apoptosis and invasion (12-14). lncRNAs are highly valuable for determining the pathological characteristics of different types of tumor, analyzing the prognosis and providing appropriate treatment (15). A number of IncRNAs, with either tumor suppressive or carcinogenic function have been identified in the past decades (16).

With regards to $\mathrm{UM}$, IncRNA is considered to play a role in regulating cell proliferation, migration and invasion, and thus, is deemed essential to the occurrence and development of UM (17-19). Small nucleolar RNA host gene 7 (SNHG7) is a recognized lncRNA, located on chromosome 9 q34.3 (20), with a total length of 2,176 base pairs (21). Previous studies have demonstrated that SNHG7 acts as a carcinogenic non-coding RNA in several types of cancer, including pancreatic (22), colorectal (20), bladder (23), gastric (24) and breast cancer (25), whereby it facilitates the proliferation, migration and invasion of tumor cells. In addition, enhancer of zeste homolog $2(\mathrm{EZH} 2)$ is the catalytic subunit of polycomb repressive complex 2 and is associated with several types of tumor, 
including UM $(26,27)$. Previous studies have demonstrated that lncRNAs play significant roles in different types of tumor via EZH2 $(28,29)$. However, the role of SNHG7 in the development of UM and the association between SNHG7 and EZH2 have not yet been investigated.

The present study detected the expression of SNHG7 in UM and hypothesized that SNHG7 may be associated with UM prognosis via EZH2. Further functional experiments were performed by upregulating SNHG7. The expression of EZH2 was detected in MEL270 and OMM2.5 cell lines overexpressing SNHG7. The results of the present study suggest that SNHG7 may play a significant role in UM development.

\section{Materials and methods}

The cancer genome atlas (TCGA) dataset analysis. Detailed SNHG7 mRNA expression data and clinical information of 80 patients with UM were obtained from TCGA database (https://portal.gdc.cancer.gov/). Patients were grouped according to median SNHG7 expression level (cutoff value $=73.695$ FPKM). The overall survival (OS) analysis and correlation analysis of SNHG7 and EZH2 were obtained from the Gene Expression Profiling Interactive Analysis (GEPIA) database (http://gepia.cancer-pku.cn) (30). The association between SNHG7 and clinical staging, as well as between EZH2 and clinical staging and histology type were obtained from the UALCAN data-mining platform (http://ualcan.path. uab.edu) (31).

Cell lines and patient tissues. A total of six human UM cell lines (92.1, MEL202, MEL270, MEL290, OMM2.3 and OMM2.5) were provided by WuXi AppTec (https://www.wuxiapptec. com/zh-cn). The 92.1, MEL202, MEL270 and MEL290 cell lines derived from non-metastatic tissues, while the OMM2.3 and OMM2.5 cell lines derived from metastatic tissues. UM cell lines were cultured in RPMI 1640 medium (Gibco; Thermo Fisher Scientific, Inc.), supplemented with $10 \%$ fetal bovine serum (FBS; Gibco; Thermo Fisher Scientific, Inc.) and 1\% penicillin-streptomycin (Beyotime Institute of Biotechnology), at $37^{\circ} \mathrm{C}$ with $5 \% \mathrm{CO}_{2}$. A total of seven $\mathrm{UM}$ tissue samples were acquired from the tissue bank of Eye \& ENT Hospital of Fudan University (Shanghai, China). The seven participants included 2 men and 5 women, with a mean age of 52 years (range, 24-71). Of these, three patients did not develop tumor metastasis, while four patients did develop metastasis. The present study was approved by the Ethics Committee of The Eye \& ENT Hospital of Fudan University, and all procedures agreed with The Declaration of Helsinki. All patients provided written informed consent prior to treatment.

Transfection. A lentiviral vector containing human IncRNA SNHG7 and an empty lentiviral vector were purchased from Genomeditech Co., Ltd. MEL270 and OMM2.5 cells were transfected with lentiviral vectors as follows: Cells were plated in 24 -well plates and incubated overnight at $37^{\circ} \mathrm{C}$. Virus solution ( $4 \times 10^{5} \mathrm{TU} /$ well; Genomeditech Co., Ltd.) and polybrene $(5 \mu \mathrm{g} / \mathrm{ml}$; Genomeditech Co., Ltd.) were added to the cells after $24 \mathrm{~h}$. Following $16 \mathrm{~h}$ of transfection, the lentiviral-containing medium was replaced with RPMI 1640 medium supplemented with $10 \%$ FBS. After $72 \mathrm{~h}$, puromycin
( $2 \mu \mathrm{g} / \mathrm{ml}$; Genomeditech Co., Ltd.) was added to the culture medium and changes in gene expression were evaluated via reverse transcription-quantitative (RT-q)PCR.

RT-qPCR analysis. Total RNA of six human UM cell lines (92.1, MEL202, MEL270, MEL290, OMM2.3 and OMM2.5) and seven UM tissue samples was extracted using TRIzol ${ }^{\circledR}$ reagent (Invitrogen; Thermo Fisher Scientific, Inc.) and reverse transcribed into cDNA using the PrimeScript ${ }^{\mathrm{TM}}$ RT Reagent kit (Takara Biotechnology Co., Ltd.). qPCR was subsequently performed using the SYBR ${ }^{\circledR}$ Green qPCR kit (Takara Biotechnology Co., Ltd.), on the ViiA ${ }^{\text {Ti }} 7$ Real-Time PCR System (Applied Biosystems; Thermo Fisher Scientific, Inc., Waltham, MA, USA). The following thermocycling conditions were used for PCR: Initial denaturation at $95^{\circ} \mathrm{C}$ for $30 \mathrm{sec} ; 40$ cycles of $95^{\circ} \mathrm{C}$ for $5 \mathrm{sec}$ and $60^{\circ} \mathrm{C}$ (annealing and extension) for $34 \mathrm{sec}$, according to the manufacturer's protocol (SYBR ${ }^{\circledR}$ Green qPCR kit; Takara Biotechnology Co.,Ltd.). The following primer sequences were used for PCR: SNHG7: Forward, 5'-TTGCTGGCGTCTCGGTTA AT-3' and reverse, 5'-GGAAGTCCATCACAGGCGAA-3'; GAPDH: Forward, 5'-TGTTGCCATCAATGACCCCTT-3' and reverse, 5'-CTCAGCCTTGACGGTGCCAT-3'; $\beta$-actin: Forward, 5'-CATGTACGTTGCTATCCAGGC-3' and reverse, 5'-CTCCTTAATGTCACGCACGAT-3'; U1: Forward, 5'-GAC GGGAAAAGATTGAGCGG-3' and reverse, 5'-GCCACG AAGAGAGTCTTGAAGG-3'. The relative mRNA levels were calculated using the $2^{-\Delta \Delta \mathrm{Cq}}$ method (32) and normalized to the internal reference gene GAPDH.

Cell counting kit-8 (CCK-8) assay. The Cell Counting Kit-8 assay was performed, according to the manufacturer's protocol (CCK-8; Dojindo Molecular Technologies, Inc.). UM cells were cultured in 96-well plates at a density of 2,000 cells/well for 4 days, at $37^{\circ} \mathrm{C}$ with $5 \% \mathrm{CO}_{2}$. CCK-8 solution $(10 \mu \mathrm{l} /$ well $)$ was added prior to incubation at $37^{\circ} \mathrm{C}$ for $2 \mathrm{~h}$. Cell viability was subsequently analyzed at a wavelength of $450 \mathrm{~nm}$, using a microplate reader (Tecan Group, Ltd.).

Flow cytometric analysis. The apoptosis assay was performed using the Annexin-V-FITC apoptosis kit (BD Biosciences). Cells were trypsinised, collected and washed twice with pre-cooled PBS. A cell suspension $\left(1 \times 10^{6}\right.$ cells $\left./ \mathrm{ml}\right)$ was prepared with $1 \mathrm{x}$ Binding buffer (BD Biosciences), and $100 \mu \mathrm{l}$ of the solution was added to each tube. Annexin V (5 $\mu \mathrm{l})$ and Propidium Iodide $(5 \mu \mathrm{l})$ (BD Biosciences) were added and mixed manually. The sample was placed in the dark for $15 \mathrm{~min}$ at $25^{\circ} \mathrm{C}$ and $1 \mathrm{x}$ Binding Buffer (400 $\mu \mathrm{l}$; BD Biosciences) was added to each sample. Apoptotic cells were subsequently analyzed via flow cytometry, using MoFlo XDP (Beckman Coulter, Inc.).

For the cell cycle analysis, the cells were harvested in a tube and washed twice with $4 \mathrm{ml}$ PBS. Pre-cooled $75 \%$ ethanol was added and incubated overnight at $4^{\circ} \mathrm{C}$. The cells were washed twice to remove all ethanol. Cell staining was performed using $1 \times 10^{6}$ cells for each tube sample. For PI/RNase staining, the cells were resuspended in $0.5 \mathrm{ml} \mathrm{PI} / \mathrm{RNase}$ staining solution (BD Biosciences) and incubated for $15 \mathrm{~min}$ at $25^{\circ} \mathrm{C}$ in the dark. The sample was stored at $4^{\circ} \mathrm{C}$ in the dark before analysis. Flow cytometric analysis was performed within $1 \mathrm{~h}$. 
Table I. SNHG7 expression and clinicopathological features of 80 patients with uveal melanoma from The Cancer Genome Atlas database.

\begin{tabular}{|c|c|c|c|c|c|}
\hline \multirow[b]{2}{*}{ Characteristics } & \multirow[b]{2}{*}{ Number of cases, $n$} & \multicolumn{2}{|c|}{ SNHG7 expression } & \multirow[b]{2}{*}{$\chi^{2}$} & \multirow[b]{2}{*}{ P-value } \\
\hline & & High & Low & & \\
\hline Sex & & & & 0.051 & 0.822 \\
\hline Male & 45 & 22 & 23 & & \\
\hline Female & 35 & 18 & 17 & & \\
\hline Tumor basal diameter, mm & & & & 1.602 & 0.206 \\
\hline$\leq 16$ & 34 & 20 & 14 & & \\
\hline$>16$ & 45 & 20 & 25 & & \\
\hline Tumor thickness, mm & & & & 1.257 & 0.262 \\
\hline$\leq 10$ & 37 & 21 & 16 & & \\
\hline$>10$ & 43 & 19 & 24 & & \\
\hline Extrascleral extension & & & & 2.891 & 0.089 \\
\hline No & 68 & 38 & 30 & & \\
\hline Yes & 7 & 1 & 6 & & \\
\hline Histological type & & & & 10.026 & 0.002 \\
\hline Epithelioid cell & 34 & 10 & 24 & & \\
\hline Spindle Cell & 46 & 30 & 16 & & \\
\hline Tumor status & & & & 6.872 & 0.009 \\
\hline Tumor-free & 61 & 35 & 26 & & \\
\hline Tumor & 18 & 4 & 14 & & \\
\hline Pathological T & & & & 1.385 & 0.239 \\
\hline II & 14 & 9 & 5 & & \\
\hline III\&IV & 66 & 31 & 35 & & \\
\hline Pathological N & & & & N.A. & N.A. \\
\hline NO & 52 & 27 & 25 & & \\
\hline NX \& null & 28 & 13 & 15 & & \\
\hline Pathological M & & & & 2.311 & 0.128 \\
\hline M0 & 51 & 27 & 24 & & \\
\hline M1+ & 4 & 0 & 4 & & \\
\hline Vital status & & & & 4.501 & 0.034 \\
\hline Alive & 67 & 37 & 30 & & \\
\hline Dead & 13 & 3 & 10 & & \\
\hline
\end{tabular}

M1+, M1a/M1b/M1c; NX, nearby (regional) lymph nodes cannot be evaluated; Null, data cannot be obtained; N.A., not applicable; T, Tumor; $\mathrm{N}$, Node; M, Metastasis.

Western blotting. Total protein of MEL270 and OMM2.5 was extracted using RIPA buffer with proteinase inhibitor (Beyotime Institute of Biotechnology). Total protein was measured using a BCA assay. Equal amounts of protein (20 $\mu \mathrm{g} /$ lane) were separated via $12 \%$ SDS-PAGE and subsequently transferred onto a polyvinylidene difluoride membrane. The membranes were blocked using $5 \% \mathrm{BSA}$, at $25^{\circ} \mathrm{C}$ for $2 \mathrm{~h}$. and subsequently incubated with the following primary antibodies, overnight at $4^{\circ} \mathrm{C}$ : Specific monoclonal EZH2 (1:1,000; cat. no. 5246) and GAPDH (1:1,000; cat. no. 2118), both from Cell Signaling Technology, Inc. Membranes were washed three times with Tris-buffered saline Tween 20 buffer (TBST; $10 \mathrm{mM}$ Tris, $150 \mathrm{mM} \mathrm{NaCl}, 0.05 \%$ Tween-20; Beijing Solarbio Science \& Technology Co., Ltd). Following the primary incubation, membranes were incubated with the horseradish peroxidase-conjugated (HRP) anti-rabbit secondary antibody (1:5,000; cat. no. 7074; Cell Signaling Technology, Inc.) at $25^{\circ} \mathrm{C}$ for $2 \mathrm{~h}$. Membranes were re-washed three times with TBST buffer. Electrochemiluminescence (cat. no. 6883; Cell Signaling Technology, Inc.) was used for visualization of the protein bands. GAPDH was used as the loading control and protein expression was quantified using ImageJ Software version 1.47 (National Institutes of Health) (33).

Subcellular fractionation. The MEL270 and OMM2.5 cell lines were divided into nuclear and cytoplasmic fractions to extract RNA prior to RT-qPCR, in order to determine the cell localization of SNHG7 by using the PARIS kit (Thermo Fisher 
A

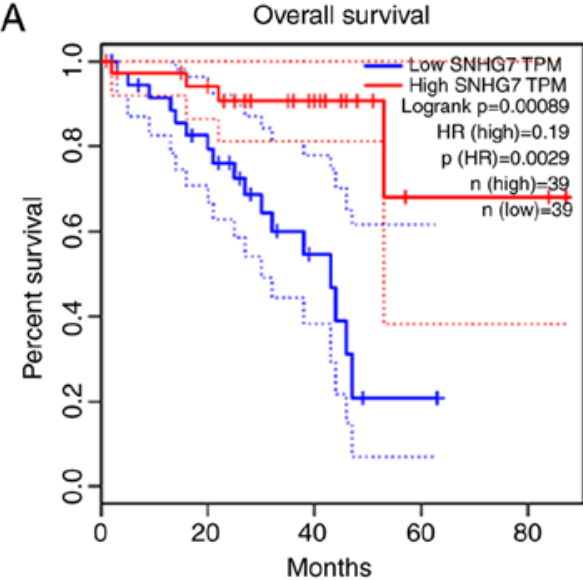

B

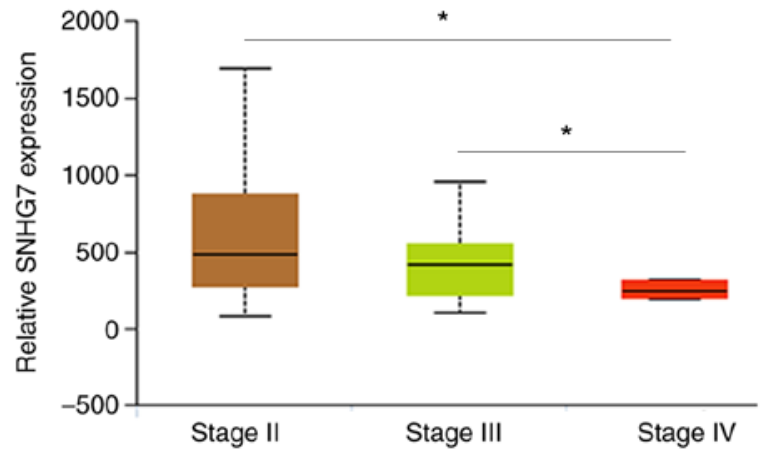

Figure 1. Low SNHG7 expression is associated with poor prognosis in UM. (A) OS analysis suggested that low SNHG7 expression was associated with significantly poor OS rate. (B) Low SNHG7 expression was associated with poor clinical staging in UM. *P<0.05 vs. stage IV group. SNHG7, small nucleolar RNA host gene 7; UM, uveal melanoma; OS, overall survival.

Scientific, Inc.). U1 (nucleus control) and $\beta$-actin (cytoplasm control) were used for normalization. Subcellular localizations of SNHG7 in 15 cell lines were obtained from LncATLAS (http://ncatlas.crg.eu).

Statistical analysis. Statistical analysis was performed using GraphPad Prism version 5.0 (GraphPad Software, Inc.). The data are presented as the mean \pm standard deviation. The mean of two independent samples was compared using Student's t-test. The associations between SNHG7 mRNA level and clinical pathological parameters of the UM specimens were assessed using $\chi^{2}$ test. Correlation analysis and survival curve analyses were performed using Pearson's correlation analysis and the Kaplan-Meier method respectively, through the GEPIA database. $\mathrm{P}<0.05$ was considered to indicate a statistically significant difference.

\section{Results}

Low expression of SNHG7 in UM is associated with poor prognosis. The public database, TCGA was used for in-depth analysis of UM. Clinical information and SNHG7 mRNA expression data were downloaded from 80 UM cases in TCGA (Table I). Patients were divided into two groups (low- and-high expression groups), according to the median expression level of SNHG7 (cutoff value=73.695 FPKM). The results of the present study suggest that low expression of SNHG7 was associated with a higher proportion of epithelioid cells $(\mathrm{P}=0.002$; Table I), fewer cases of tumor-free survival ( $\mathrm{P}=0.009$; Table I) and a higher death rate $(\mathrm{P}=0.034$; Table I) The histological types of UM included epithelioid and spindle, which accounted for 42.5 and $57.5 \%$ of total cases, respectively. There were 61 tumor-free cases $(76.25 \%)$ and 18 cases $(22.5 \%)$ with tumor. A total of 67 cases $(83.75 \%)$ were alive whereas 13 cases $(16.25 \%)$ were dead. Furthermore, the OS analysis suggested that lower SNHG7 expression is significantly associated with poor OS compared with higher SNHG7 expression in patients with UM $(\mathrm{P}<0.05$; Fig. 1A). The present study analyzed the results of TCGA database using the UALCAN platform and confirmed that SNHG7 is associated with clinical staging (Stage II vs.
Stage IV, $\mathrm{P}<0.05$; Stage III vs. Stage IV, $\mathrm{P}<0.05$; Fig. 1B). The results of the present study demonstrated that downregulated expression of SNHG7 was associated with poor prognosis in patients with UM.

SNHG7 expression in UM tissues and six UM cell lines. In order to further determine the biological functions of SNHG7 in the development of UM, the present study investigated the differential expression of SNHG7 in UM tissues of patients both with and without metastasis, using RT-qPCR. SNHG7 expression in UM was significantly downregulated in the metastatic group compared with the non-metastatic group $(\mathrm{P}<0.001$; Fig. 2A). Furthermore, SNHG7 expression levels in the six UM cell lines were analyzed using RT-qPCR, and the cell lines with low expression of SNHG7 were selected for the SNHG7 overexpression experiment (Fig. 2B). The results of the present study demonstrated that the expression levels of SNHG7 in the cell lines derived from metastasis (OMM2.5) were relatively low. Among the cell lines derived from non-metastasis (92.1, MEL202, MEL270 and MEL290), MEL270 exhibited the lowest SNHG7 expression levels. Thus, the present study selected the OMM2.5 cell line derived from metastasis, and the MEL270 cell line derived from non-metastasis for further investigation. Subcellular localizations of SNHG7 in 15 cell lines were obtained from lncATLAS (34), in order to determine the association between cell localization and lncRNA function. SNHG7 was predominantly expressed in the cytoplasm in HepG2 (liver cancer cell line) and A549 (lung cancer cell line); however, SNHG7 was predominantly expressed in the nucleus in K562 (human leukemia cell line) (Fig. 2C). The present study performed subcellular fractionation in order to detect the localization of SNHG7 in UM cell lines. MEL270 and OMM2.5 cell lines were divided into nuclear and cytoplasmic fractions. Subsequently, RT-qPCR was performed in order to identify the subcellular localization of SNHG7, which confirmed that SNHG7 was preferentially located in the nucleus in both MEL270 and OMM2.5 cell lines (Fig. 2D and E).

Upregulation of SNHG7 inhibits cell proliferation in UM cell lines. SNHG7 expression levels were significantly upregulated 

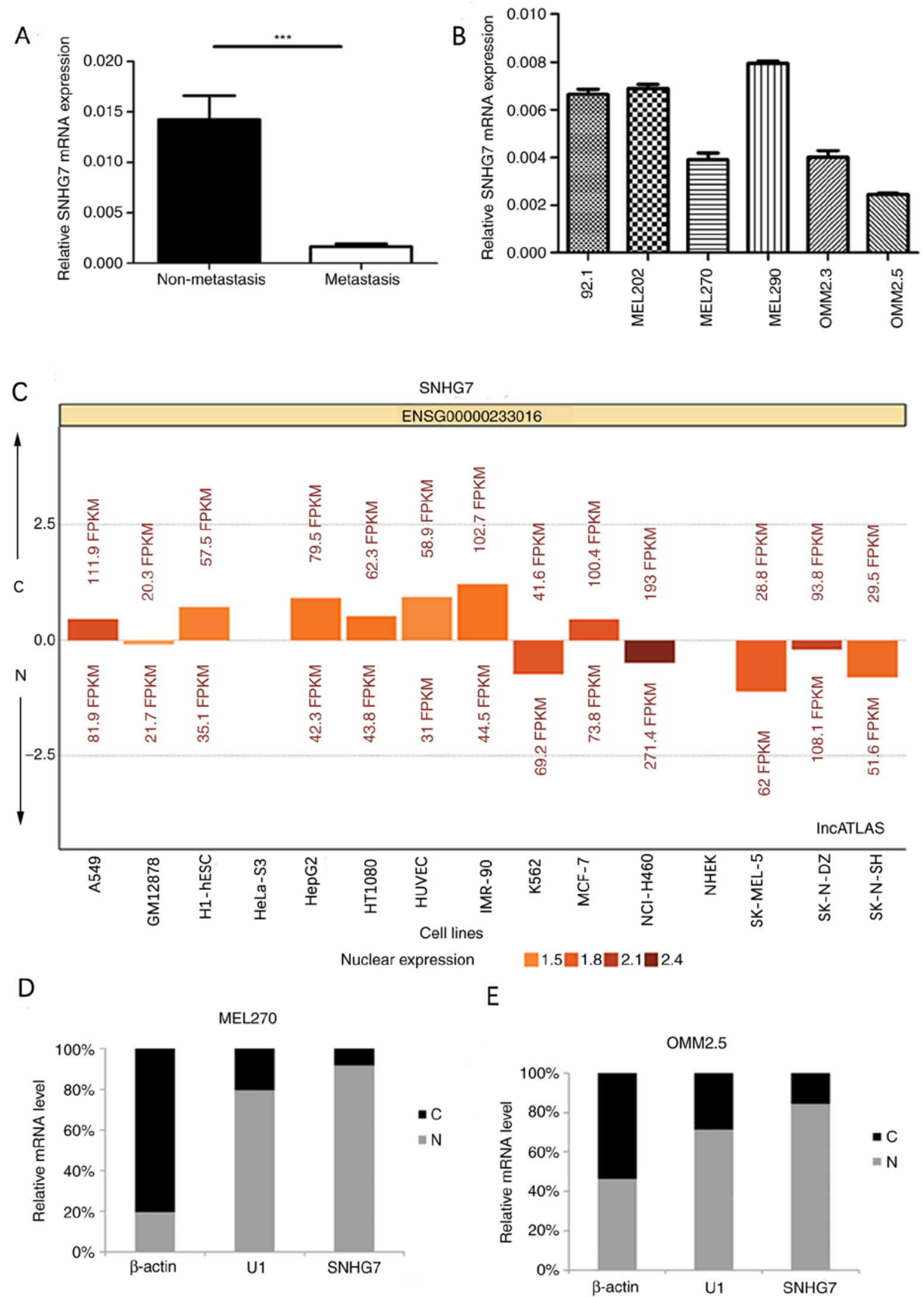

Figure 2. Reverse transcription-quantitative PCR analysis of SNHG7 expression levels in UM tissues and cell lines. (A) Relative SNHG7 mRNA expression levels in tissues of patients with UM. SNHG7 levels were significantly downregulated in patients with distant metastasis compared with patients without distant metastasis. (B) SNHG7 expression levels were measured in six UM cell lines (92.1, MEL202, MEL270, MEL290, OMM2.3 and OMM2.5). The MEL270 and OMM2.5 cell lines were selected for further analyses. (C) Subcellular localization of SNHG7 in 15 cell lines (A549, GM12878, H1-hESC, HeLa-S3, HepG2, HT1080, HUVEC, IMR-90, K562, MCF-7, NCI-H460, NHEK, SK-MEL-5, SK-N-DZ and SK-N-SH). (D) Localization of SNHG7 in MEL270 and (E) OMM2.5 cell lines. SNHG7 was preferentially located in the nucleus in both MEL270 and OMM2.5 cell lines. $\beta$-actin and U1 were used as cytoplasmic and nuclear site markers, respectively. ${ }^{* * *} \mathrm{P}<0.001$ vs. non-metastasis group. SNHG7, small nucleolar RNA host gene 7; UM, uveal melanoma; C, cytoplasm; $\mathrm{N}$, nucleus. 

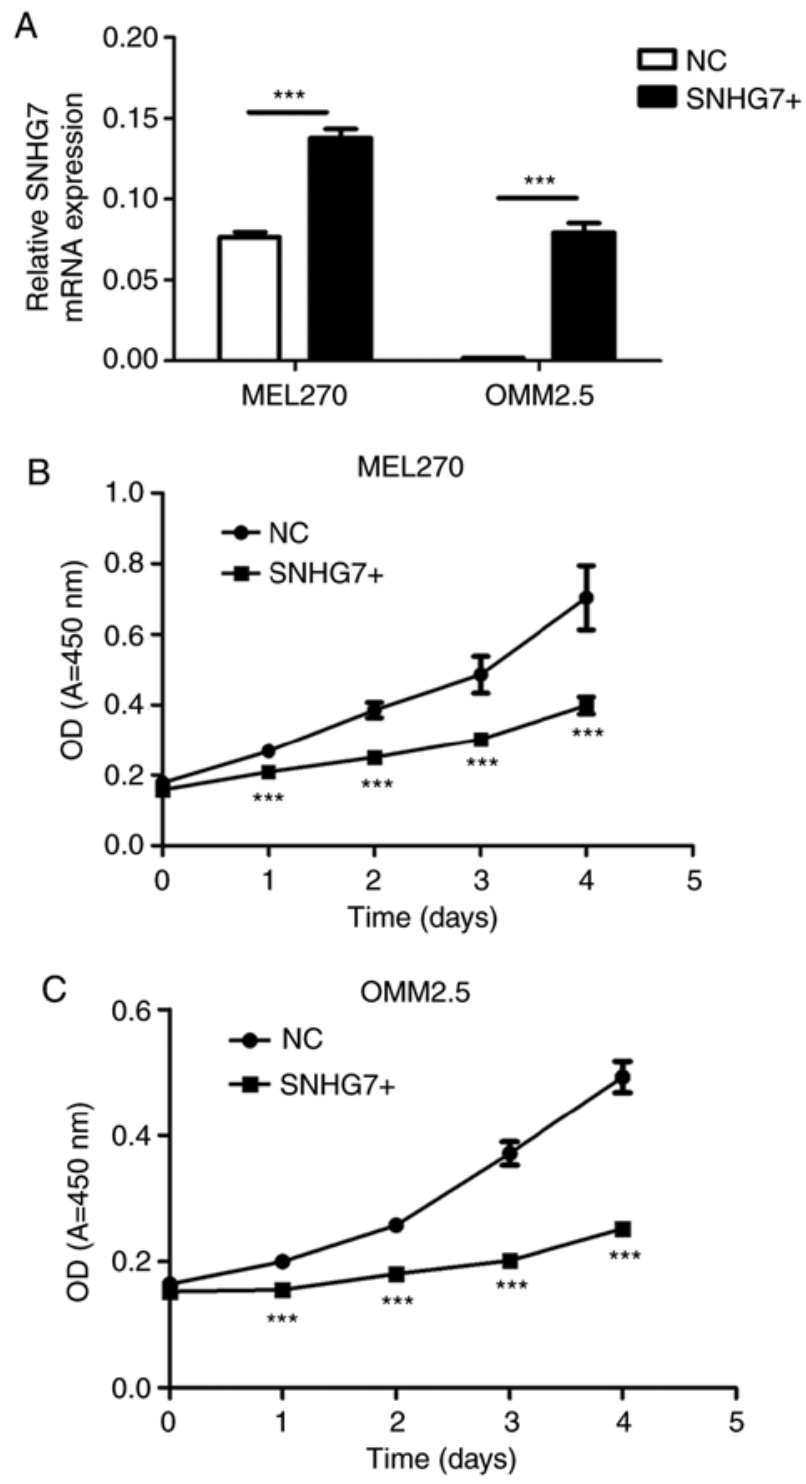

Figure 3. Overexpression of SNHG7 suppresses cell proliferation in both MEL270 and OMM2.5 cell lines. (A) SNHG7 expression levels were measured via reverse transcription-quantitative PCR in the SNHG7+ and NC groups in both MEL270 and OMM2.5 cell lines. (B) Cell proliferation of MEL270 and (C) OMM2.5 cell lines was measured via Cell Counting Kit- 8 assays. Overexpression of SNHG7 suppressed cell proliferation of UM cells in both MEL270 and OMM2.5 cell lines. ${ }^{* * *} \mathrm{P}<0.001$ vs. NC group. SNHG7, small nucleolar RNA host gene 7; NC, empty vector group; UM, uveal melanoma; OD, optical density; A, absorbance.

by lentivirus infection in both the MEL270 and OMM2.5 cell lines, and the overexpression efficiency was detected via RT-qPCR ( $\mathrm{P}<0.001$; Fig. 3A). As presented in Fig. 3B and C, overexpression of SNHG7 in the MEL270 and OMM2.5 cell lines significantly inhibited cell proliferation. The cells overexpressing SNHG7 in both MEL270 and OMM2.5 cell lines demonstrated a significant decrease in cell proliferation following incubation for 4 days $(\mathrm{P}<0.001$; Fig. 3B and $\mathrm{C})$. The results of the present study demonstrated that SNHG7 significantly inhibited the proliferation of UM cells.

Overexpression of SNHG7 in UM cell lines induces cell cycle arrest and promotes apoptosis in vitro. In order to investigate the effect of SNHG7 overexpression on cell cycle, the present study performed a cell cycle analysis to determine whether SNHG7 also inhibited cell cycle progression. The results of the present study suggest that overexpression of SNHG7 could affect the cell cycle of both MEL270 and OMM2.5 cell lines. The proportion of overexpressed SNHG7 cells in $\mathrm{G}_{0} / \mathrm{G}_{1}$ phase significantly increased, with a decline in $\mathrm{S}$ phase in both MEL270 ( $\mathrm{P}<0.05$; Fig. 4A-D) and OMM2.5 ( $\mathrm{P}<0.001$; Fig. 4E-H) cell lines. The results of the present study indicate that SNHG7 imposes a strong blocking effect on UM cell cycle.

The apoptotic rate (early + late stage apoptosis) in the SNHG7+ and the NC groups, in both MEL270 and OMM2.5 cell lines was measured using the Annexin-V-FITC apoptosis kit, in order to detect whether SNHG7 exerted anticancer effects on UM cells. The results of the present study demonstrated that the apoptotic rate in the MEL270 SNHG7+ group was $3.530 \pm 0.241 \%$ compared with the MEL270 NC group (3.090 $\pm 0.092 \%, \mathrm{P}<0.05$; Fig. 5A, B and E), the OMM2.5 SNHG7+ group $(3.47 \pm 0.315 \%)$ and the OMM2.5 NC group $(0.72 \pm 0.147 \%, \mathrm{P}<0.001$; Fig. 5C-E), and the differences were statistically significant (Fig. 5E). The results of the present study indicate that overexpression of SNHG7 promotes apoptosis of UM cells.

SNHG7 suppresses UM cell line proliferation, induces cell cycle arrest and promotes apoptosis by inhibiting EZH2. It is well known that lncRNA can bind specific proteins in order to exert its molecular function (35). Correlation analysis in the present study indicated that SNHG7 and EZH2 were correlated ( $\mathrm{R}=-0.41, \mathrm{P}=0.00019$; Fig. 6A). The OS analysis demonstrated that a higher EZH2 expression level was associated with a poor OS; however, the difference was not significant between the high and low EZH2 expression levels ( $\mathrm{P}>0.05$; Fig. 6B). Conversely, EZH2 was demonstrated to be associated with clinical staging (stage III vs. stage IV, $\mathrm{P}<0.05$; Fig. 6C) and histological type $(\mathrm{P}<0.05$; Fig. 6D).

The present study detected EZH2 expression at the protein level via western blot analysis. Overexpression of SNHG7 was demonstrated to have an inhibitory effect on EZH2. The SNHG7 overexpression group of MEL270 and OMM2.5 cell lines had a lower expression of EZH2 compared with the NC groups $(\mathrm{P}<0.01$; Fig. 6E). The results of the present study indicate that overexpression of SNHG7 inhibits EZH2 expression, suggesting that EZH2 may be a downstream target of SNHG7.

\section{Discussion}

$\mathrm{UM}$ is the most common intraocular tumor in adults worldwide (36). UM has been widely studied; however, the molecular mechanism underlying its occurrence and development remains unknown. Thus, investigating the pathogenesis of UM may aid in the development of novel therapeutic targets. A number of lncRNAs are involved in the development and progression of UM, as oncogenes or tumor suppressors (37). For example, FTH1P3 promotes the proliferation and migration of UM cells by inhibiting the expression of miR-224-5p (17). Furthermore, the lncRNA PAUPAR inhibits the occurrence of UM by preventing $\mathrm{H} 3 \mathrm{~K} 4$ demethylation, which exerts a tumor suppressing effect (38). Although a large number of lncRNAs have been demonstrated to be associated with UM, numerous IncRNAs have not yet been discovered. In the present study, 

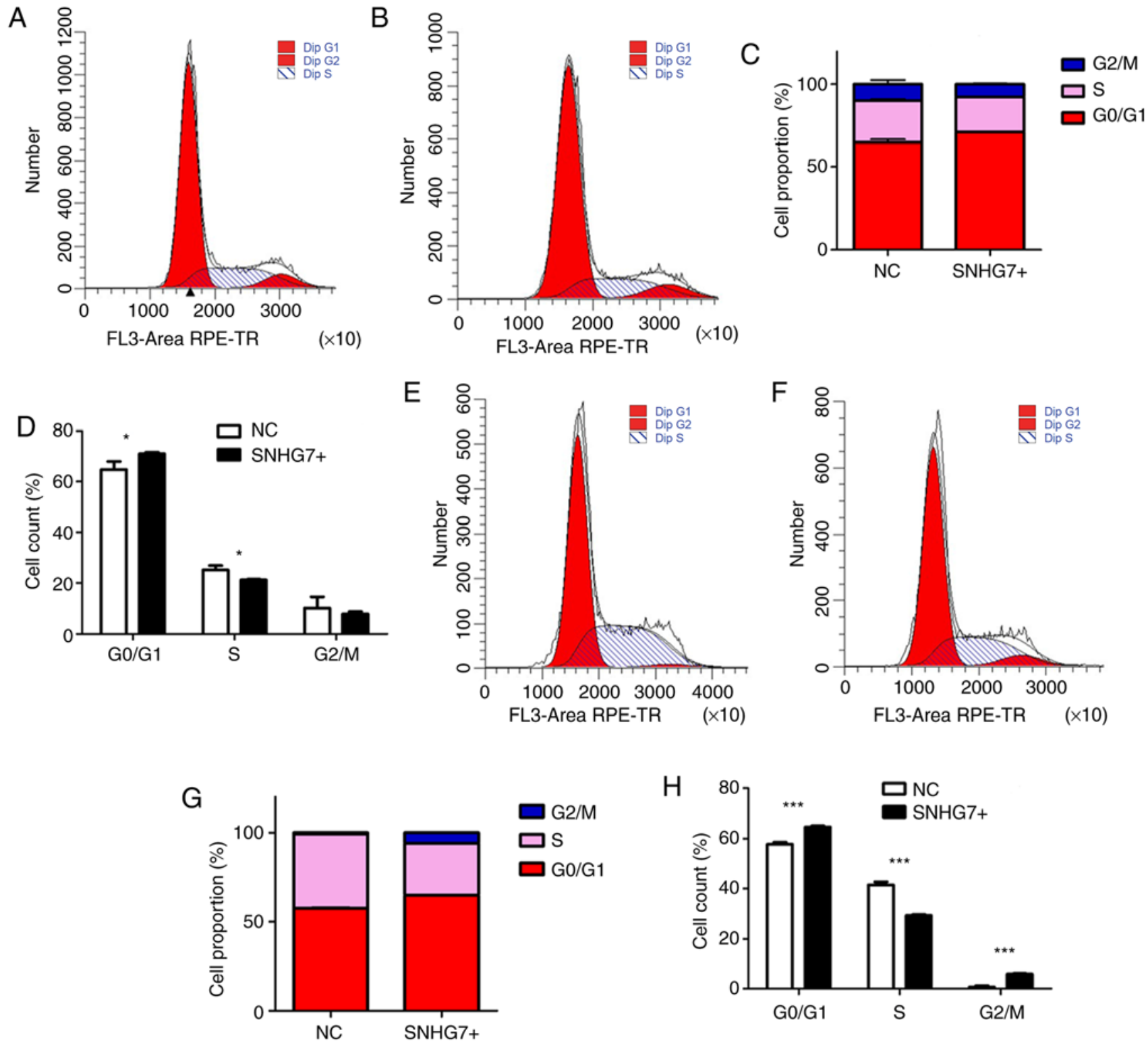

Figure 4. Effects of SNHG7 overexpression on cell cycle in both MEL270 and OMM2.5 cell lines. Cell cycle progression analyses of MEL270 cell line in both (A) NC and (B) SNHG7+ groups. (C) Distribution of each phase in both groups of MEL270 cell line. (D) Statistical analysis of cell cycle phases in MEL270 cell line. Cell cycle progression analyses of OMM2.5 cell line in both (E) NC and (F) SNHG7+ groups. (G) Distribution of each phase in both groups of OMM2.5 cell line. $(\mathrm{H})$ Statistical analysis of cell cycle phases in OMM2.5 cell line. The proportion in $\mathrm{G}_{0} / \mathrm{G}_{1}$ phase significantly increased, with a decline in $\mathrm{S}$ phase in both MEL270 and OMM2.5 cell lines. ${ }^{*} \mathrm{P}<0.05,{ }^{* * *} \mathrm{P}<0.001$ vs. NC group. SNHG7, small nucleolar RNA host gene 7; NC, empty vector group.

80 clinical UM specimens from TCGA database were analyzed. The OS analysis indicated that low expression of SNHG7 resulted in a significantly lower OS rate. Furthermore, the expression of SNHG7 was significantly associated with histological types, tumor-free survival and vital status. The present study set out to investigate the function and underlying molecular mechanism of SNHG7 in UM, and demonstrated that overexpression of SNHG7 significantly suppressed the proliferation and cell cycle, while promoting apoptosis in both MEL270 and OMM2.5 cell lines.

SNHG7 has previously been studied as an oncogene $(20,22,23)$. However, a number of lncRNAs play a dual role, namely, oncogenes or tumor suppressors in different types of cancer. For example, ZFAS1 plays a tumor-suppressor role in breast cancer; however, it acts as an oncogene in non-small cell lung, colorectal, gastric, liver, ovarian and bladder cancer (39).
Furthermore, the lncRNA, H19 has also been reported to have either anti-cancer or carcinogenic effects in different types of tumor $(40,41)$. As research progresses, the role of SNHG7 in different types of tumor may gradually be confirmed. The present study demonstrated that SNHG7 played a role in inhibiting malignant transformation in UM. SNHG7 inhibited cell proliferation, induced cell cycle arrest and promoted apoptosis. Contradictory to a previous study (42), SNHG7 was preferentially located in the nucleus in both MEL270 and OMM2.5 cell lines in the present study. UM is significantly different from cutaneous melanoma and has unique tumorigenic processes and tumor biology (43). Thus, the present study suggests that the effect of SNHG7 in UM may be attributed to the specificity of the intraocular tumor.

EZH2 serves a significant role in human carcinomas (44-46) and regulates the processes of cell proliferation, cell cycle 
A

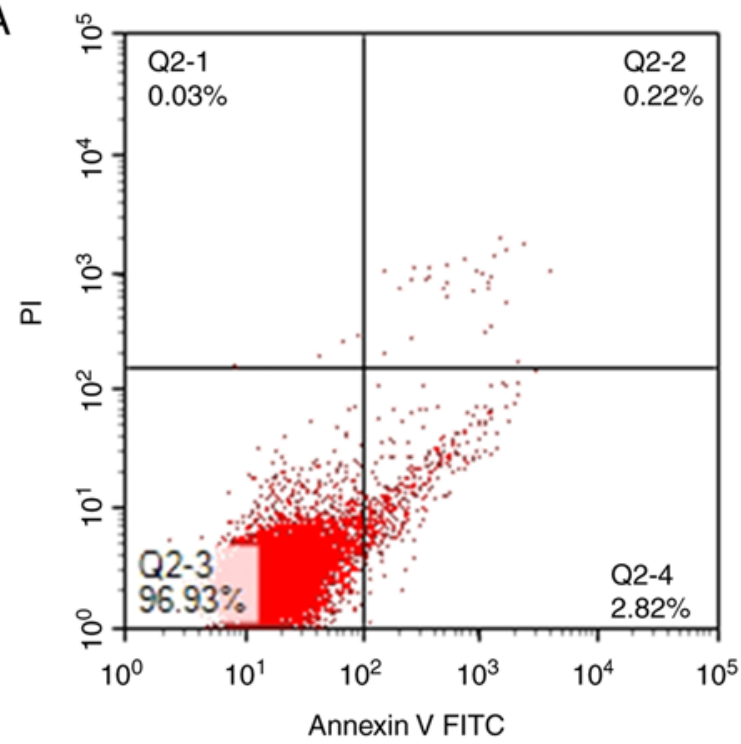

C

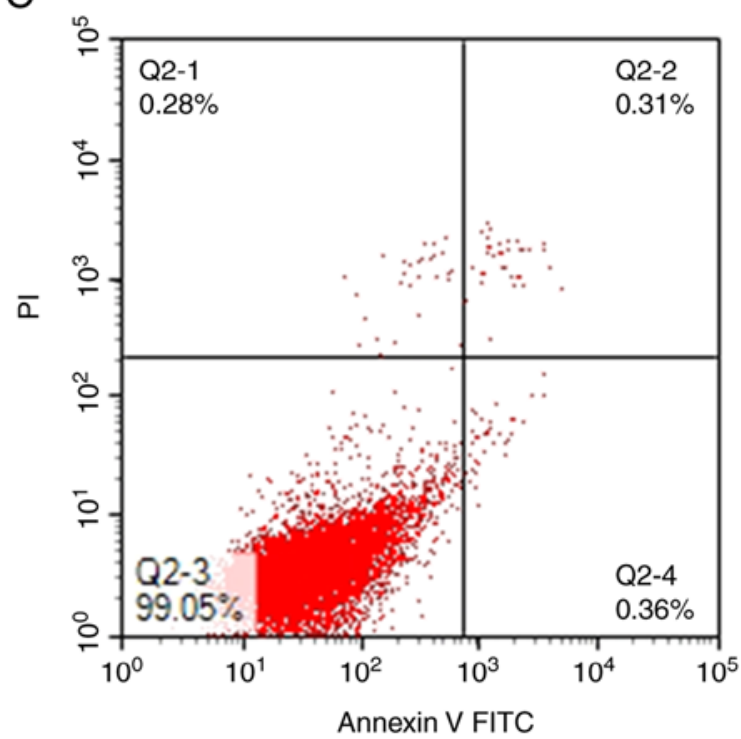

B

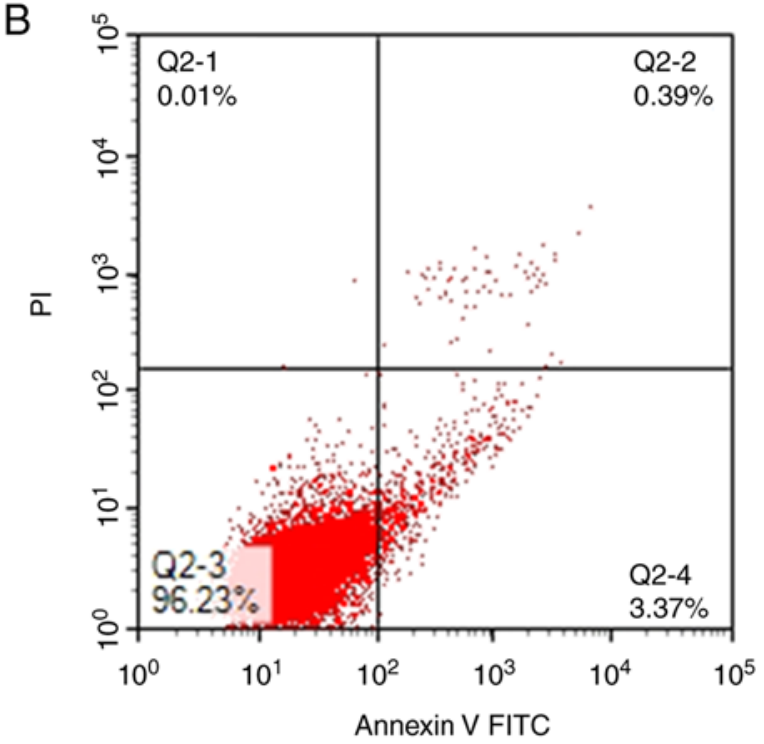

D

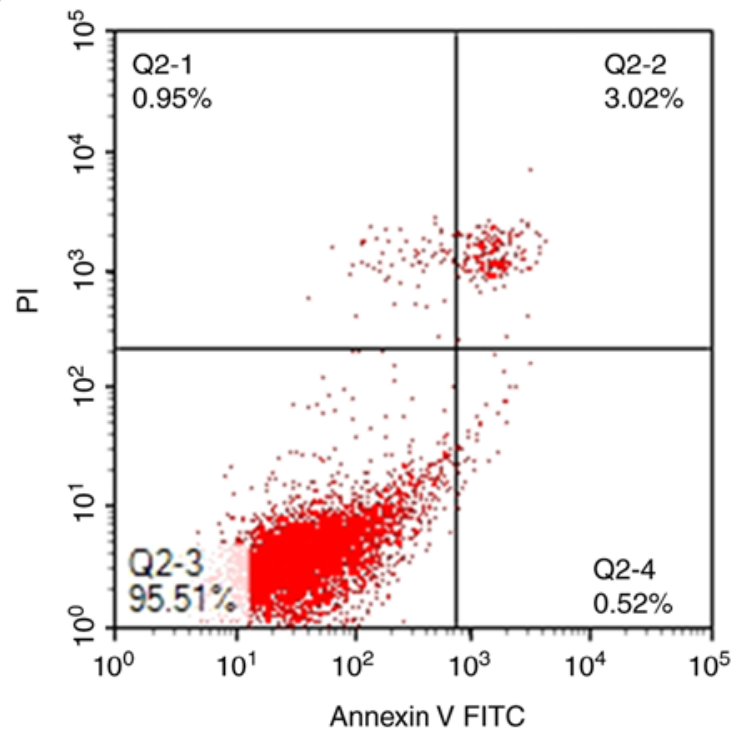

E

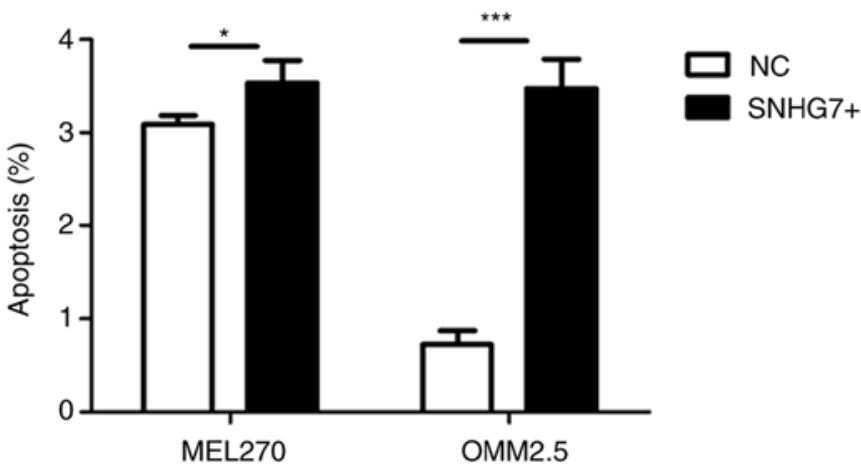

Figure 5. Apoptotic effect of SNHG7 overexpression on both MEL270 and OMM2.5 cell lines. (A-D) The effect of SNHG7 overexpression on cell apoptosis in MEL270 and OMM2.5 cell lines was measured via flow cytometry. The results suggested that upregulation of SNHG7 induced apoptosis compared with the NC group (A: MEL270 NC group; B: MEL270 SNHG7+ group; C: OMM2.5 NC group and D: OMM2.5 SNHG7+ group). (E) The data are presented as mean \pm standard deviation of three independent experiments. ${ }^{*} \mathrm{P}<0.05,{ }^{* * *} \mathrm{P}<0.001$ vs. NC group. SNHG7, small nucleolar RNA host gene 7; NC, empty vector group; PI, propidium iodide; Annexin V-FITC, Annexin V-Fluorescein isothiocyanate.

and apoptosis (47-49). Notably, EZH2 is involved in the malignant transformation of different types of tumor in both cutaneous melanoma and UM $(27,50)$. In the present study,
EZH2 expression was demonstrated to be associated with SNHG7. Higher EZH2 expression levels were associated with a higher Tumor-Node-Metastasis (TNM) stage (51) and a poor 
A

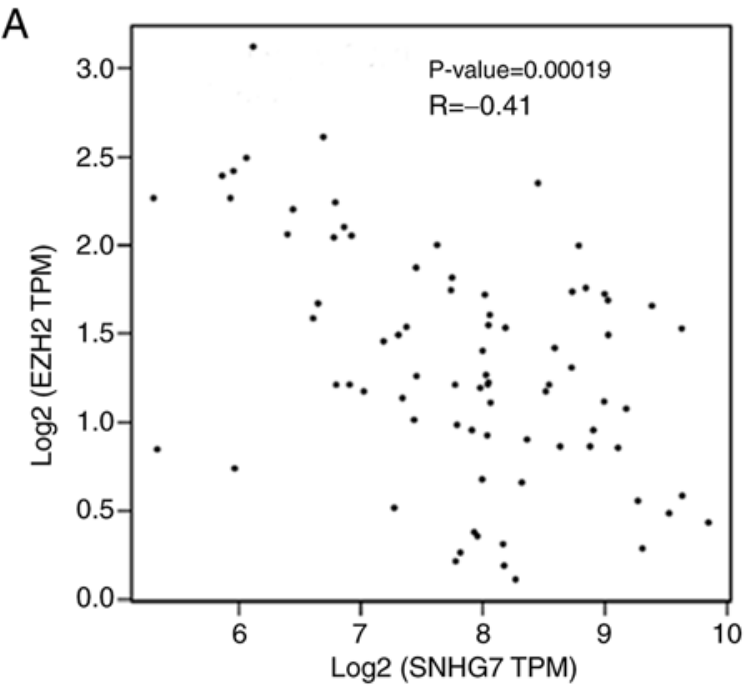

B

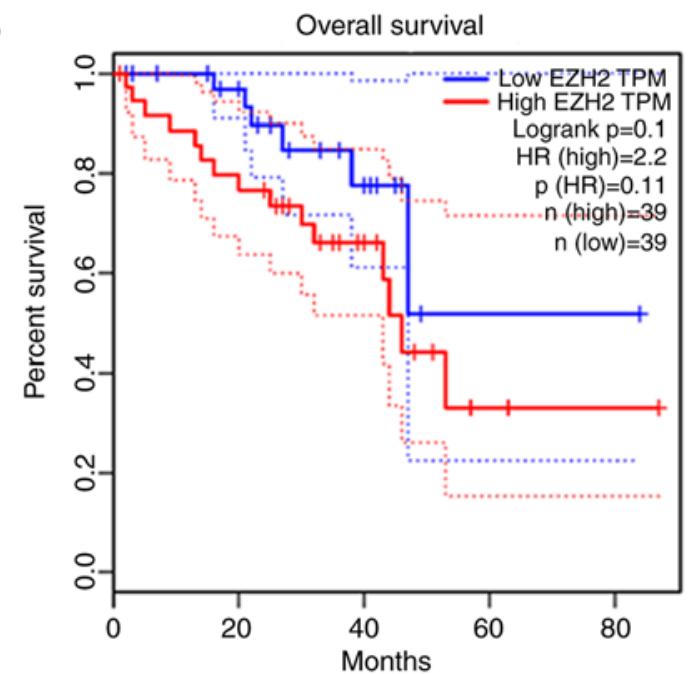

D

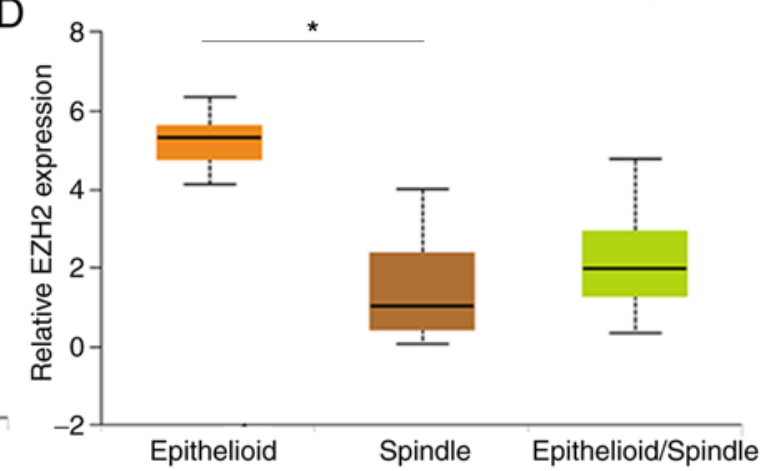

Epithelioid
$\mathrm{E}$

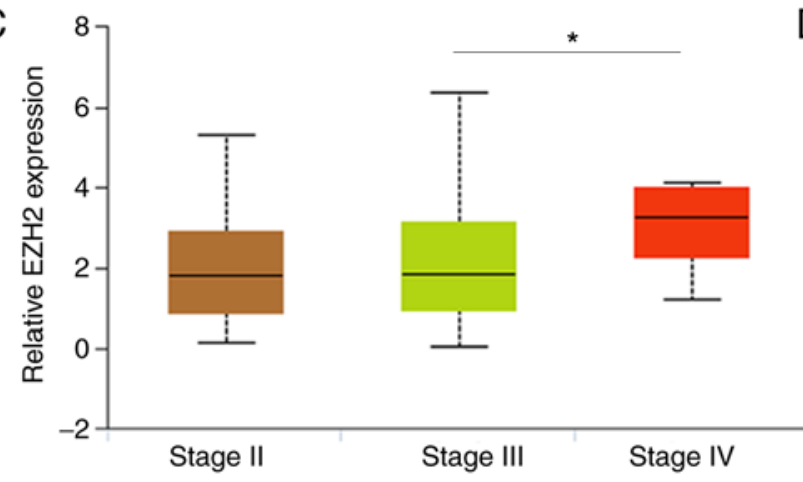

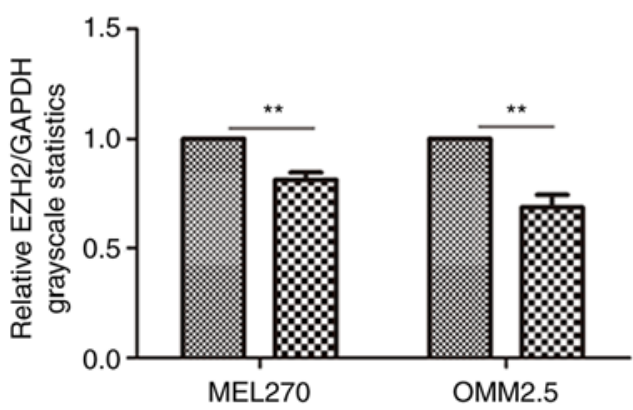

Figure 6. EZH2 is downregulated in SNHG7 overexpressed cells. (A) SNHG7 and EZH2 were correlated. The expression of EZH2 decreased as the expression of SNHG7 increased. (B) OS analysis of EZH2 in UM. High EZH2 expression level was associated with a poor OS, but the difference was not significant between the high and low EZH2 expression groups. EZH2 was associated with (C) poor clinical staging and (D) histological type. (E) Western blot analysis of EZH2 was performed. There was lower protein expression levels of EZH2 while SNHG7 was upregulated in both MEL270 and OMM2.5 cell lines. "P<0.05 vs. stage IV group; ${ }^{*} \mathrm{P}<0.05$ vs. Spindle cell group; ${ }^{* *} \mathrm{P}<0.01$ vs. NC group. EZH2, enhancer of zeste homolog 2; SNHG7, small nucleolar RNA host gene 7; OS, overall survival; UM, uveal melanoma; $\mathrm{NC}$, empty vector group.

histological type. In addition, the present study demonstrated that SNHG7 inhibited EZH2 expression. This suggests that SNHG7 could inhibit the proliferation, mediate cell cycle and induce apoptosis in UM cells, and its molecular mechanism may be associated with the inhibition of EZH2.

Overall, the present study demonstrated that SNHG7 played a vital role in UM via EZH2. Although numerous studies have been performed on UM, there are currently no effective treatments for UM, particularly for high-risk patients. The development of appropriate treatment is of great importance in order to improve the survival rate of patients with UM. The present study set out to investigate the molecular mechanism underlying UM development. The results of the present study suggest that SNHG7 may be a potential novel target for the diagnosis and treatment of UM. However, further experiments in vivo and associated clinical trials are required for verification.

\section{Acknowledgements}

The authors would like to thank Dr Yun Cheng (Department of Ophthalmology, Eye \& ENT Hospital of Fudan University, Shanghai, China) for her help in performing the experiments, and Dr Qian Wu (Department of Pathology, West China 
Hospital, Sichuan University, Sichuan, China) for providing suggestions for the revision of the manuscript.

\section{Funding}

The present study was funded by the National Natural Science Foundation of China (grant nos. 81970835 and 81800867).

\section{Availability of data and materials}

The datasets used and/or analyzed during the current study are available from the corresponding author upon reasonable request.

\section{Authors' contributions}

RZ designed and supervised the present study. XW performed the majority of the experiments and drafted the initial manuscript. YY helped design the study. RM helped perform the experiments and revised the language of the manuscript. BX interpreted the data. All authors have read and approved the final version of the manuscript.

\section{Ethics approval and consent to participate}

The present study was approved by the Institutional Review Board of the Eye \& ENT Hospital of Fudan University (Shanghai, China). All patients provided written informed consent prior to the study start.

\section{Patient consent for publication}

Not applicable.

\section{Competing interests}

The authors declare that they have no competing interests.

\section{References}

1. Chandran SS, Somerville RPT, Yang JC, Sherry RM, Klebanoff CA, Goff SL, Wunderlich JR, Danforth DN, Zlott D, Paria BC, et al: Treatment of metastatic uveal melanoma with adoptive transfer of tumour-infiltrating lymphocytes: A single-centre, two-stage, single-arm, phase 2 study. Lancet Oncol 18: 792-802, 2017.

2. Robertson AG, Shih J, Yau C, Gibb EA, Oba J, Mungall KL, Hess JM, Uzunangelov V, Walter V, Danilova L, et al: Integrative analysis identifies four molecular and clinical subsets in uveal melanoma. Cancer Cell 32: 204-220.e15, 2017.

3. Field MG, Durante MA, Anbunathan H, Cai LZ, Decatur CL, Bowcock AM, Kurtenbach S and Harbour JW: Punctuated evolution of canonical genomic aberrations in uveal melanoma. Nat Commun 9: 116, 2018

4. Lane AM, Kim IK and Gragoudas ES: Survival rates in patients after treatment for metastasis from uveal melanoma. JAMA Ophthalmol 136: 981-986, 2018.

5. Yoo JH, Shi DS, Grossmann AH, Sorensen LK, Tong Z Mleynek TM, Rogers A, Zhu W, Richards JR, Winter JM, et al: ARF6 is an actionable node that orchestrates oncogenic GNAQ signaling in uveal melanoma. Cancer Cell 29: 889-904, 2016.

6. Batista PJ and Chang HY: Long noncoding RNAs: Cellular address codes in development and disease. Cell 152: 1298-1307, 2013.

7. Liu Y, Zhang R, Qiu F, Li K, Zhou Y, Shang D and Xu Y: Construction of a lncRNA-PCG bipartite network and identification of cancer-related lncRNAs: A case study in prostate cancer. Mol Biosyst 11: 384-393, 2015.
8. Ye B, Liu B, Yang L, Zhu X, Zhang D, Wu W, Zhu P, Wang Y, Wang S, Xia P, et al: LncKdm2b controls self-renewal of embryonic stem cells via activating expression of transcription factor Zbtb3. EMBO J 37: e97174, 2018

9. Zhang L, Meng X, Zhu XW, Yang DC, Chen R, Jiang Y and $\mathrm{Xu}$ T: Long non-coding RNAs in Oral squamous cell carcinoma: Biologic function, mechanisms and clinical implications. Mol Cancer 18: 102, 2019.

10. Martens-Uzunova ES, Bottcher R, Croce CM, Jenster G, Visakorpi T and Calin GA: Long noncoding RNA in prostate, bladder, and kidney cancer. Eur Urol 65: 1140-1151, 2014.

11. Parasramka MA, Maji S, Matsuda A, Yan IK and Patel T: Long non-coding RNAs as novel targets for therapy in hepatocellular carcinoma. Pharmacol Ther 161: 67-78, 2016.

12. Wang G, Zhang ZJ, Jian WG, Liu PH, Xue W, Wang TD, Meng YY, Yuan C, Li HM, Yu YP, et al: Novel long noncoding RNA OTUD6B-AS1 indicates poor prognosis and inhibits clear cell renal cell carcinoma proliferation via the $\mathrm{Wnt} / \beta$-catenin signaling pathway. Mol Cancer 18: 15, 2019.

13. Murugan AK, Munirajan AK and Alzahrani AS: Long noncoding RNAs: Emerging players in thyroid cancer pathogenesis. Endocr Relat Cancer 25: R59-R82, 2018.

14. Huang JL, Zheng L, Hu YW and Wang Q: Characteristics of long non-coding RNA and its relation to hepatocellular carcinoma. Carcinogenesis 35: 507-514, 2014.

15. Wang FW, Cao CH, Han K, Zhao YX, Cai MY, Xiang ZC, Zhang JX, Chen JW, Zhong LP, Huang Y, et al: APC-activated long noncoding RNA inhibits colorectal carcinoma pathogenesis through reduction of exosome production. J Clin Invest 129: 727-743, 2019.

16. Noh JH and Gorospe M: AKTions by cytoplasmic lncRNA CASC9 promote hepatocellular carcinoma survival. Hepatology 68: 1675-1677, 2018.

17. Zheng X, Tang H, Zhao X, Sun Y, Jiang Y and Liu Y: Long non-coding RNA FTH1P3 facilitates uveal melanoma cell growth and invasion through miR-224-5p. PLoS One 12: e0184746, 2017.

18. Lu Q, Zhao N, Zha G, Wang H, Tong Q and Xin S: LncRNA HOXA11-AS exerts oncogenic functions by repressing p21 and miR-124 in uveal melanoma. DNA Cell Biol 36: 837-844, 2017.

19. Lu L, Yu X, Zhang L, Ding X, Pan H, Wen X, Xu S, Xing Y, Fan J, $\mathrm{Ge} \mathrm{S}$, et al: The long non-coding RNA RHPN1-AS1 promotes uveal melanoma progression. Int J Mol Sci 18: pii: E226, 2017.

20. Li Y, Zeng C, Hu J, Pan Y, Shan Y, Liu B and Jia L: Long non-coding RNA-SNHG7 acts as a target of miR-34a to increase GALNT7 level and regulate PI3K/Akt/mTOR pathway in colorectal cancer progression. J Hematol Oncol 11: 89, 2018.

21. Ota T, Suzuki Y, Nishikawa T, Otsuki T, Sugiyama T, Irie R, Wakamatsu A, Hayashi K, Sato H, Nagai K, et al: Complete sequencing and characterization of 21,243 full-length human cDNAs. Nat Genet 36: 40-45, 2004.

22. Cheng D, Fan J, Ma Y, Zhou Y, Qin K, Shi M and Yang J: LncRNA SNHG7 promotes pancreatic cancer proliferation through ID4 by sponging miR-342-3p. Cell Biosci 9: 28, 2019.

23. Zhong X, Long Z, Wu S, Xiao M and Hu W: LncRNA-SNHG7 regulates proliferation, apoptosis and invasion of bladder cancer cells assurance guidelines. J BUON 23: 776-781, 2018.

24. Wang MW, Liu J, Liu Q, Xu QH, Li TF, Jin S and Xia TS: LncRNA SNHG7 promotes the proliferation and inhibits apoptosis of gastric cancer cells by repressing the P15 and P16 expression. Eur Rev Med Pharmacol Sci 21: 4613-4622, 2017.

25. Luo X, Song Y, Tang L, Sun DH and Ji DG: LncRNA SNHG7 promotes development of breast cancer by regulating microRNA-186. Eur Rev Med Pharmacol Sci 22: 7788-7797, 2018.

26. Villanueva MT: Anticancer drugs: All roads lead to EZH2 inhibition. Nat Rev Drug Discov 16: 239, 2017.

27. Huang XM, Shi SS, Jian TM, Tang DR, Wu T and Sun FY: LncRNA PVT1 knockdown affects proliferation and apoptosis of uveal melanoma cells by inhibiting EZH2. Eur Rev Med Pharmacol Sci 23: 2880-2887, 2019.

28. Li Z, Hou P, Fan D, Dong M, Ma M, Li H, Yao R, Li Y, Wang G, Geng P, et al: The degradation of EZH2 mediated by lncRNA ANCR attenuated the invasion and metastasis of breast cancer. Cell Death Differ 24: 59-71, 2017.

29. Jia B, Xie T, Qiu X, Sun X, Chen J, Huang Z, Zheng X, Wang Z and Zhao J: Long noncoding RNA FALEC inhibits proliferation and metastasis of tongue squamous cell carcinoma by epigenetically silencing ECM1 through EZH2. Aging (Albany NY) 11: 4990-5007, 2019 . 
30. Tang Z, Li C, Kang B, Gao G, Li C and Zhang Z: GEPIA: A web server for cancer and normal gene expression profiling and interactive analyses. Nucleic Acids Res 45 (W1): W98-W102, 2017.

31. Chandrashekar DS, Bashel B, Balasubramanya SAH Creighton CJ, Ponce-Rodriguez I, Chakravarthi BVSK and Varambally S: UALCAN: A portal for facilitating tumor subgroup gene expression and survival analyses. Neoplasia 19: 649-658, 2017

32. Livak KJ and Schmittgen TD: Analysis of relative gene expression data using real-time quantitative PCR and the 2(-Delta Delta C(T)) method. Methods 25: 402-408, 2001.

33. Schneider CA, Rasband WS and Eliceiri KW: NIH image to Image J: 25 years of image analysis. Nat Methods 9: 671-675, 2012.

34. Mas-Ponte D, Carlevaro-Fita J, Palumbo E, Hermoso Pulido T, Guigo R and Johnson R: LncATLAS database for subcellular localization of long noncoding RNAs. RNA 23: 1080-1087, 2017.

35. Chaudhary $R$ and Lal A: Long noncoding RNAs in the p53 network. Wiley Interdiscip Rev RNA 8, 2017.

36. Van Raamsdonk CD, Griewank KG, Crosby MB, Garrido MC Vemula S, Wiesner T, Obenauf AC, Wackernagel W, Green G, Bouvier N, et al: Mutations in GNA11 in uveal melanoma. N Engl J Med 363: 2191-2199, 2010.

37. Sun J, Pan LM, Chen LB and Wang Y: LncRNA XIST promotes human lung adenocarcinoma cells to cisplatin resistance via let-7i/BAG-1 axis. Cell Cycle 16: 2100-2107, 2017.

38. Ding X, Wang X, Lin M, Xing Y, Ge S, Jia R, Zhang H, Fan X and Li J: PAUPAR IncRNA suppresses tumourigenesis by H3K4 demethylation in uveal melanoma. FEBS Lett 590: 1729-1738, 2016.

39. Jiang $X$, Yang $Z$ and Li Z: Zinc finger antisense 1: A long noncoding RNA with complex roles in human cancers. Gene 688 26-33, 2019.

40. Lan X, Sun W, Dong W, Wang Z, Zhang T, He L, Zhang H: Downregulation of long noncoding RNA H19 contributes to the proliferation and migration of papillary thyroid carcinoma. Gene 646: 98-105, 2018.

41. Liang WQ, Zeng, Chen CF, Sun SM, Lu XF, Peng CY and Lin HY: Long noncoding RNA H19 is a critical oncogenic driver and contributes to epithelial-mesenchymal transition in papillary thyroid carcinoma. Cancer Manag Res 11: 2059-2072, 2019.

42. Shan Y, Ma J, Pan Y, Hu J, Liu B and Jia L: LncRNA SNHG7 sponges miR-216b to promote proliferation and liver metastasis of colorectal cancer through upregulating GALNT1. Cell Death Dis 9: 722, 2018.
43. Pandiani C, Beranger GE, Leclerc J, Ballotti R and Bertolotto C: Focus on cutaneous and uveal melanoma specificities. Genes Dev 31: 724-743, 2017.

44. Kim KH and Roberts CW: Targeting EZH2 in cancer. Nat Med 22: 128-134, 2016.

45. McCabe MT, Ott HM, Ganji G, Korenchuk S, Thompson C, Van Aller GS, Liu Y, Graves AP, Della Pietra A III, Diaz E, et al: EZH2 inhibition as a therapeutic strategy for lymphoma with EZH2-activating mutations. Nature 492: 108-112, 2012.

46. Varambally S, Dhanasekaran SM, Zhou M, Barrette TR, Kumar-Sinha C, Sanda MG, Ghosh D, Pienta KJ, Sewalt RG, Otte AP, et al: The polycomb group protein EZH2 is involved in progression of prostate cancer. Nature 419: 624-629, 2002.

47. Zingg D, Debbache J, Schaefer SM, Tuncer E, Frommel SC, Cheng P, Arenas-Ramirez N, Haeusel J, Zhang Y, Bonalli M, et al: The epigenetic modifier EZH2 controls melanoma growth and metastasis through silencing of distinct tumour suppressors. Nat Commun 6: 6051, 2015

48. Chen S, Pu J, Bai J, Yin Y, Wu K, Wang J, Shuai X, Gao J, Tao K, Wang $\mathrm{G}$ and $\mathrm{Li} \mathrm{H}$ : EZH2 promotes hepatocellular carcinoma progression through modulating miR-22/galectin-9 axis. J Exp Clin Cancer Res 37: 3, 2018.

49. Hubaux R, Thu KL, Coe BP, MacAulay C, Lam S and Lam WL: EZH2 promotes E2F-driven SCLC tumorigenesis through modulation of apoptosis and cell-cycle regulation. $\mathbf{J}$ Thorac Oncol 8: 1102-1106, 2013.

50. Bachmann IM, Halvorsen OJ, Collett K, Stefansson IM, Straume O, Haukaas SA, Salvesen HB, Otte AP and Akslen LA: EZH2 expression is associated with high proliferation rate and aggressive tumor subgroups in cutaneous melanoma and cancers of the endometrium, prostate, and breast. J Clin Oncol 24: 268-273, 2006

51. Kivelä T and Kujala E: Prognostication in eye cancer: The latest tumor, node, metastasis classification and beyond. Eye (Lond) 27 : $243-252,2013$

This work is licensed under a Creative Commons Attribution-NonCommercial-NoDerivatives 4.0 International (CC BY-NC-ND 4.0) License. 